\title{
Capacity of Intelligent Quadrifiliar Helix Antenna (IQHA) Based MIMO System
}

\author{
Yinan Qi, Reza Hoshyar, Rahim Tafazolli \\ Centre for Communication System Research, \\ University of Surrey, Guildford, Surrey, UK GU2 7XH \\ Email: $\underline{\text { Y.Qi@surrey.ac.uk }}$
}

\begin{abstract}
We investigate the capacity of Intelligent Quadrifiliar Helix Antenna (IQHA) based Multiple-inputmultiple-output (MIMO) communication system. We will show that IQHA based MIMO system is able to offer larger capacity compared with MIMO system without using IQHA. And at the same time, it can reduce the number of RF chains following the antenna, thus reducing the total cost. Two sub-optimal algorithms are proposed to adjust the weights of IQHA to maximize the capacity.
\end{abstract}

INDEX TERMS: IQHA, adaptive antenna array, MIMO, capacity, Radio Frequency (RF), Base Band (BB)

\section{INTRODUCTION}

In recent years, MIMO communication system characterized as multiple transmitting and receiving antennas has draw more and more attentions because it is able to offer higher capacity [1][2]. However, it increases the processing complexity and hardware cost significantly. Each branch requires a RF chain including upconverter/down-converter, $\mathrm{D} / \mathrm{A}$ or $\mathrm{A} / \mathrm{D}$ and power amplifier and so on. Although the antennas and digital signal processing are cheap, the RF element are expensive and do not follow Moore's law [6], [7]. Therefore, the need to decrease the number of RF chains requires us to decrease the number of chains while keeping the system performance unchanged. Application of IQHA with weight adjusting in MIMO system may achieve this goal. An IQHA based MIMO communication system will be introduced in this paper and the capacity improvement will be discussed. This paper is organized as following. Section II is a brief introduction of IQHA structure. In Section III, system model and capacity analysis will be presented and two sub-optimisation Algorithms are introduced. Section IV includes simulation results and discussion. Conclusion will be made in last section.

\section{IQHA STRUCTURE}

IQHA consists of three main parts: the antennas structure itself, the adaptive components (weight factors, matching circuits and switches) and the overall control part [3]-[5].The matching circuits and switches allow antenna to operate in multiple frequencies. And the controller adjusts the weight factors to maximise the chosen criterion. In this paper, since the multiple frequencies operability has no influence on the following discussion, the structure can be simplified in Fig. 1.

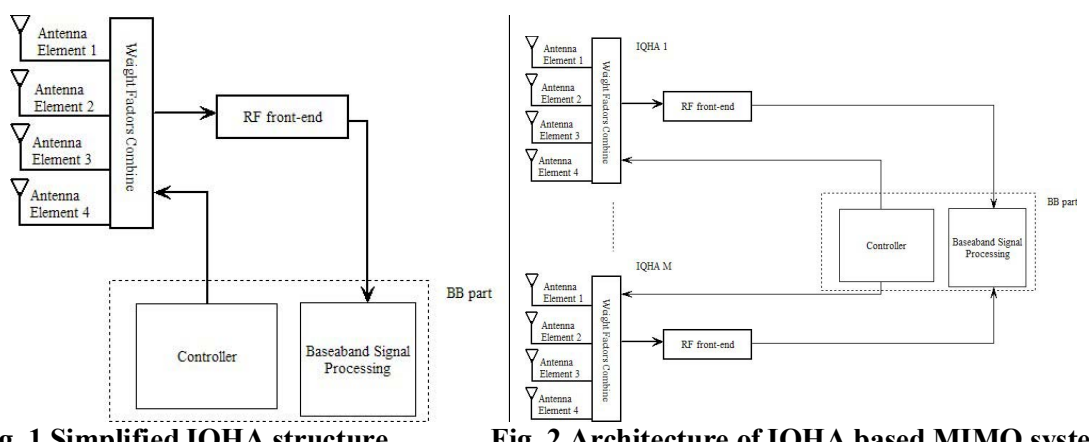

Fig. 1 Simplified IQHA structure

Fig. 2 Architecture of IQHA based MIMO system

In Fig. 1, the received signal is combined with weight factors that are adjusted by the controller in Base Band (BB) and processed in BB signal processor after RF chains.

\section{SYSTEM MODEL AND ANALYSIS}

Previously, IQHA was used in SIMO to achieve the spatial diversity [5]. And the potential of IQHA in MIMO system is also discussed in [9]. However, in all of these applications, there is only one IQHA in the receiver end. Different from previous applications, each branch of the IQHA based MIMO system is an IQHA. That means multiple IQHA will be applied in our system as shown in Fig. 2. 


\section{A. New Capacity Equation}

Suppose $\mathrm{N} * \mathrm{M}$ MIMO system and $\mathrm{J}$ antenna elements in IQHA, the receiving antennas are M IQHAs and each IQHA has $\mathrm{J}=4$ antennas elements. As we know, the noise consists of two main parts: noise from channel (channel instability, cosmic radiation, etc.) and noise produced by the RF chains. Therefore, we divide noise into two parts. The received signal is given by

$r=H s+n_{i n} \dashv n_{r f}$

$n_{i n}$ : Noise from channel $n_{r f}$ : Noise from RF chains

We make two assumptions here. First, both $n_{i n}$ and $n_{r f}$ have independent Gaussian-distributed elements with equal variance. Second, $n_{i n}$ and $n_{r f}$ are independent. So suppose the variance of $n$ is $C^{2}$, the variance of $n_{i n}$ is $\rho \sigma^{2}$ and the variance of $n_{r f}$ is $(1-\rho) \sigma^{2}$.The received signal after weights adjusting is:

$r=\sum_{j=1}^{J}\left(H_{j} s+n_{i n}^{j}\right)+n_{r f}=H s+\sum_{j=1}^{J} n_{i n}^{j}+n_{r f}$

$s=\left[s_{1}(t), \cdots, s_{N}(t)\right]^{T}, r=\left[r_{1}(t), \cdots, r_{M}(t)\right]^{T}, n_{i n}^{j}=\left[w_{1, j} n_{i n}^{j 1}(t), \cdots, w_{M, j} n_{i n}^{j M}(t)\right]^{T}, n_{r f}=\left[n_{r f}^{1}(t), \cdots, n_{r f}^{M}(t)\right]^{T}$

$w=\left[\begin{array}{lll}w_{1,1} & \cdots & w_{1, J} \\ \vdots & \ddots & \vdots \\ w_{M, 1} & \cdots & w_{M, J}\end{array}\right], H_{j}=\left[\begin{array}{lll}w_{1, j} h_{11}^{j} & \cdots & w_{1, j} h_{1 N}^{j} \\ \vdots & \ddots & \vdots \\ w_{M, j} h_{M 1}^{j} & \cdots & w_{M, j} h_{M N}^{j}\end{array}\right], H=\sum_{j=1}^{J} H_{j}$

where $w_{i, j}$ is the weight in the $j$ th antenna element of $i$ th IQHA.

According to [1],[2],[10], the capacity of MIMO channel is defined as

$C=I(s ; y)=H(y)-H(n) \quad H(y)=\log _{2}\left(\operatorname{det}\left|\pi e R_{y y}\right|\right) b p s / H z, H(n)=\log _{2}\left(\operatorname{det}\left|\pi e R_{n n}\right|\right) b p s / H z$

Here I(s;y) is the mutual information between vectors s and y. $R_{y y}=E\left\{y y^{H}\right\}$ is the covariance matrix of $\mathrm{y}$. It satisfies. If the power is equally divided in $\mathrm{N}$ transmitting antennas and the element of $\mathrm{s}$ is independent and

$R_{n n}=E\left\{n n^{T}\right\}=E\left\{\left(\sum_{j=1}^{J} n_{i n, j}+n_{r f}\right)\left(\sum_{j=1}^{J} n_{i n, j}+n_{r f}\right)^{T}\right\}=\left(J \sigma_{i n}^{2}+\sigma_{r f}^{2}\right) I_{M}=\left(J \rho \sigma^{2}+(1-\rho) \sigma^{2}\right) I_{M}$

So Channel capacity in Eq. 3 reduces to

$C=\log _{2} \operatorname{det}\left|I_{M}+\frac{E_{s}}{N \sigma^{2}(1+(J-1) \rho)} H H^{H}\right|$

From Eq. 5, we can see the capacity depends on $\rho$. And from Eq. 2, capacity also depends on weight matrix $w$.

\section{B. Sub-optimization Algorithm}

\section{Sequential searching}

In order to improve the capacity, the weights can be adjusted to maximize Eq. 6 . That means a certain weight matrix $w$ in Eq. 2 should be found to maximize the channel capacity C. The optimal solution requires not only changing the phase but also changing the amplitude of $w$. Therefore, the complexity is quite high. However, here we only adjust the phase of $w$ and search for a sub-optimal solution. So the sequential searching is proposed as follow.

1. Set $w$ as all zero phase matrix, divide $2 \pi$ by $\mathrm{K}$ and set $k \frac{2 \pi}{K}, k=0,1, \cdots, K-1$ as the interval of phase adjusting.

2. Set $m=1$ and $j=1$.

3. Keeping the phase of other weights constant and set the phase of weight $w_{m, j}$ as $\exp \left(k \frac{2 \pi}{K}\right), k=0,1, \cdots, K-1$ sequentially and compute (7) respectively.

4. Supposing Eq. 5 is maximized when $w_{m, j}=\exp \left(l \frac{2 \pi}{K}\right)$, update $_{w_{m, j}}=\exp \left(l \frac{2 \pi}{K}\right)$.

5. If $j=J, m=m+1$ and $j=1$. If $j \neq J, j=j+1$. Go to step 3

6. If $j=J, m=M$, stop. 


\section{Trace maximizing}

Although the sequential searching algorithm is easy to understand, it requires lots of computations because in each step, the Eq. 5 has to be calculated. Therefore, we are turning to other algorithm to maximizing Eq. 5. Trace maximization is given below.

For a small $\mathcal{E}$, there is

$\operatorname{det}|I+\varepsilon A|=1+\varepsilon \operatorname{Tr}(A)+O\left(\varepsilon^{2}\right)$

So if $\frac{E_{s}}{N \sigma^{2}(1+(J-1) \rho)}$ is a small number,

$\operatorname{det}\left|I_{N}+\frac{E_{s}}{N \sigma^{2}(1+(J-1) \rho)} H^{H} H\right|=1+\frac{E_{s}}{N \sigma^{2}(1+(J-1) \rho)} \operatorname{Tr}\left(H^{H} H\right)+O\left(\left(\frac{E_{s}}{N \sigma^{2}(1+(J-1) \rho)}\right)^{2}\right)$

Therefore, we can maximize capacity by maximizing trace of $H^{H} H$.

$\operatorname{Tr}\left(H^{H} H\right)=\operatorname{Tr}\left(\left(\sum_{j=1}^{J} H_{j}^{H}\right)\left(\sum_{k=1}^{J} H_{k}\right)\right)$

Since the diagonal elements of $H_{j}^{H} H_{k}$ is independent of weights $w$ if $j=k$, we only consider $j \neq k$.

$\operatorname{Tr}\left(H^{H} H\right)=\operatorname{Tr}\left(\sum_{\substack { j=1 \\ \begin{subarray}{c}{k=1 \\ k \neq j{ j = 1 \\ \begin{subarray} { c } { k = 1 \\ k \neq j } }\end{subarray}}^{J} H_{j}^{H} H_{k}\right)$

For any pair of $(j, k)$, there is another pair $(k, j)$ that is conjugate of pair $(j, k)$. Thus, we got

$$
\operatorname{Tr}\left(H^{H} H\right)=\operatorname{Tr}\left(2 \operatorname{Re}\left(\sum_{j=1}^{J} \sum_{\substack{k=1 \\
k \neq j}}^{J} H_{j}^{H} H_{k}\right)\right)=2 \operatorname{Re}\left(\operatorname{Tr}\left(\sum_{j=1}^{J} \sum_{\substack{k=1 \\
k \neq j}}^{J} H_{j}^{H} H_{k}\right)\right)=2 \operatorname{Re}\left(\begin{array}{l}
\sum_{m=1}^{M}\left(\begin{array}{l}
\exp \left(i\left(\theta_{m 2}-\theta_{m 1}\right)\right) h_{m}^{12}+\exp \left(i\left(\theta_{m 3}-\theta_{m 1}\right)\right) h_{m}^{13}+, \\
\cdots,+\exp \left(i\left(\theta_{m J}-\theta_{m 1}\right)\right) h_{m}^{1 J}
\end{array}\right) \\
\vdots \\
\left.\vdots \begin{array}{l}
M \\
\exp \left(i\left(\theta_{m 3}-\theta_{m 2}\right)\right) h_{m}^{23}+\exp \left(i\left(\theta_{m 4}-\theta_{m 2}\right)\right) h_{m}^{24}+, \\
\cdots,+\exp \left(i\left(\theta_{m J}-\theta_{m 2}\right)\right) h_{m}^{2 J}
\end{array}\right) \\
+\sum_{m=1}^{M}\left(\exp \left(i\left(\theta_{m J}-\theta_{m(J-1)}\right)\right) h_{m}^{(J-1) J}\right)
\end{array}\right)
$$

where $i=\sqrt{-1} . \theta_{m, j}$ is the phase of weight $w_{m, j}$ and $h_{m}^{j k}=\sum_{n=1}^{N} h_{m, n}^{j}{ }^{*} h_{m, n}^{k}$. In order to maximize the real part of above equation, one obvious method is to let $\theta_{m k}-\theta_{m j}=-\arg \left(h_{m}^{j k}\right) \quad$ and we can maximize the equation as $\operatorname{Tr}\left(H^{H} H\right)=\sum_{m=1}^{M} \sum_{k=j+1}^{J} \sum_{j=1}^{J}\left|h_{i}^{l k}\right|$. However, to fulfil equation, we have

$$
\begin{aligned}
& \theta_{m 2}-\theta_{m 1}=-\arg \left(h_{m}^{12}\right), \theta_{m 3}-\theta_{m 1}=-\arg \left(h_{m}^{13}\right), \cdots, \theta_{m b}-\theta_{m 1}=-\arg \left(h_{m}^{1 J}\right) \\
& \theta_{m 3}-\theta_{m 2}=-\arg \left(h_{m}^{23}\right), \cdots, \theta_{m b}-\theta_{m 2}=-\arg \left(h_{m}^{2 J}\right) \\
& \vdots \\
& \theta_{m J}-\theta_{m(J-1)}=-\arg \left(h_{m}^{(J-1) J}\right) \quad m=1,2, \ldots, M
\end{aligned}
$$

Because $\theta_{m 1}$ has no influence on these equations, we set $\theta_{m 1}=0$. It can be easily seen that if $J \leq 2$, we can get results of above equations and the trace can be maximized. But if $J>2$, those equations cannot be fulfilled at the same time. So we only fulfil the first line of the equations. And we got

$$
\theta_{m 1}=0, \theta_{m j}=-\arg \left(h_{m}^{1 j}\right) \quad m=1,2, \cdots, M \quad j=2,3, \cdots, J
$$

Therefore, we got the weights to maximize the trace of $H^{H} H$.

\section{SIMULATION RESULTS AND DISCUSSIONS}

As we can see in Fig. 3, if the power of noise from radio channel is same as the noise power from RF chains, the capacity of $2 * 2$ IQHA based MIMO system is close to or larger than the capacity of $2 * 8$ MIMO system no matter when SNR is high or low. However, the number of RF chains is only $1 / 4$ of $2 * 8$ MIMO systems. 

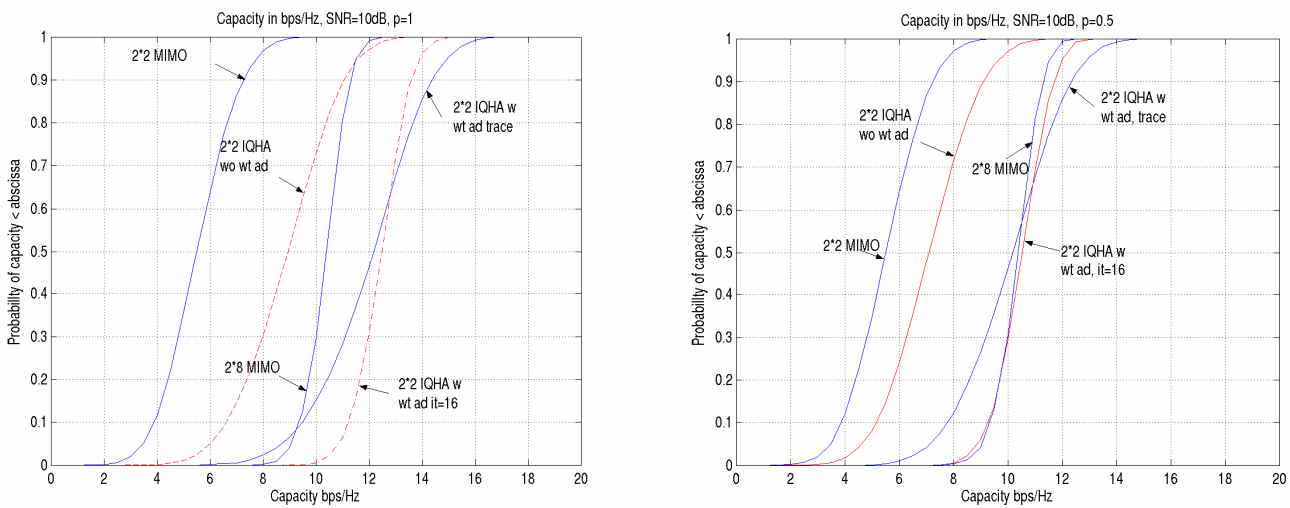

Fig 3. Capacity ( $p=1-\rho$, w: with, wo: without, wt: weight, ad: adjusting, it: sequential searching algorithm applied and $\mathrm{K}=16$, trace: trace maximization algorithm applied)

If almost all the noise is from RF chains, which happens in single-user communications [8]. The capacity of $2 * 2$ IQHA based MIMO system even has better performance in capacity compared with $2 * 8$ MIMO system with only $1 / 4$ RF chains. From figure 4 and 5, we can see that even when the $w$ is not adjusted, the capacity of $2 * 2$ IQHA based MIMO system is larger than that of normal 2*2 MIMO system.

\section{CONCLUSION}

In this paper, we investigated IQHA based MIMO system and explore the capacity performance of this system with weights adjusting. From previous discussion and simulation, it is shown that the capacity is able to be improved if the noise does not only consist of noise from channel. When the power of channel noise is same as or less than the power of noise from RF chains, compared with normal MIMO systems with same number of receiving antenna elements, the IQHA based system can achieve same or larger capacity and have less RF chains at the same time.

\section{REFERENCES}

[1] G. J. Foschini and M. J. Gans, "On limits of wireless communications in a fading environment when using multiple antennas," Wireless Personal Commun., vol. 6, pp. 311-335, Mar. 1998.

[2] Telatar, E., "Capacity of Multi-Antenna Gaussian Channels," European Transaction on Telecommunications, vol. 10, no. 6, pp. 585-595, Nov./Dec. 1999.

[3] Agius, A. A., and Saunders, S. R. "Adaptive multifilar antenna," International Patent Application No. WO9941803, Aug. 1999.

[4] S.M. Leach, A.A.Agius and S.R. Saunders, "Intelligent quadrifilar helix antenna," IEE Proc. Microw. Antenna Propag. vol. 147, no. 3, pp. 219-223, June 2000

[5] Daniel, Chew, "Practical Implementation of the Intelligent Quadrifilar Helix Antenna for Terminal Applications," PhD Thesis, University of Surrey, UK, 2004

[6] Andreas F. Molisch and Moe Z. Win, "MIMO Systems with Antenna Selection," IEEE Microwave Magazine, pp. 46-56, Mar. 2004.

[7] Shahab Sanayei and Aria nosratinia, "Antenna Selection in MIMO Systems," IEEE Communications Magazine, pp. 68-72, Oct. 2004.

[8] Jon W. Wallace and Michael A. Jensen, "Mutual Coupling in MIMO Wireless Systems: A Rigorous Network Theory Analysis," IEEE Transactions on Wireless Communications, vol. 3, no. 4, pp. 1317-1325, Jul. 2004.

[9] Daniel K. C. Chew, Ioannis Morfis, Dimitrios Mavrakis, and Stavros Stavrou, "Quadrifilar Helix Antenna for MIMO System," IEEE ANTENNAS AND WIRELESS PROPAGATION LETTERS, VOL. 3, pp. 197-199, 2004

[10] Jankiraman, Mohinder, "Space-time codes and MIMO systems," Artech House Boston · London, 2004 